\title{
Charmed baryon weak decays with vector mesons
}

\author{
C. Q. Geng, ${ }^{1,2,3,4}$ Chia-Wei Liu, ${ }^{3}$ and Tien-Hsueh Tsai ${ }^{3}$ \\ ${ }^{1}$ School of Fundamental Physics and Mathematical Sciences, Hangzhou Institute for Advanced Study, \\ UCAS, Hangzhou 310024, China \\ ${ }^{2}$ International Centre for Theoretical Physics Asia-Pacific, Beijing/Hangzhou, China \\ ${ }^{3}$ Department of Physics, National Tsing Hua University, Hsinchu 300, Taiwan \\ ${ }^{4}$ Physics Division, National Center for Theoretical Sciences, Hsinchu 300, Taiwan
}

(Received 20 January 2020; accepted 5 March 2020; published 20 March 2020)

\begin{abstract}
We give a systematic study of $\mathbf{B}_{c} \rightarrow \mathbf{B}_{n} V$ decays, where $\mathbf{B}_{c}$ and $\mathbf{B}_{n}$ correspond to the antitriplet charmed and octet baryons, respectively, while $V$ stands for the vector mesons. We calculate the color-symmetric contributions to the decays from the effective Hamiltonian with the factorization approach and extract the antisymmetric ones based on the experimental measurements and $S U(3)_{F}$ flavor symmetry. We find that most of the existing experimental data for $\mathbf{B}_{c} \rightarrow \mathbf{B}_{n} V$ are consistent with our fitting results. We present all the branching ratios of the Cabibbo allowed, singly Cabibbo suppressed, and doubly Cabibbo suppressed decays of $\mathbf{B}_{c} \rightarrow \mathbf{B}_{n} V$. The decay parameters for the daughter baryons and mesons in $\mathbf{B}_{c} \rightarrow \mathbf{B}_{n} V$ are also evaluated. In particular, we point out that the Cabibbo allowed decays of $\Lambda_{c}^{+} \rightarrow \Lambda^{0} \rho^{+}$and $\Xi_{c}^{0} \rightarrow \Xi^{-} \rho^{+}$as well as the singly Cabibbo suppressed ones of $\Lambda_{c}^{+} \rightarrow \Lambda^{0} K^{*+}, \Xi_{c}^{+} \rightarrow \Sigma^{+} \phi$, and $\Xi_{c}^{0} \rightarrow \Xi^{-} K^{*+}$ have large branching ratios and decay parameters with small uncertainties, which can be tested by the experimental searches at the charm facilities.
\end{abstract}

DOI: 10.1103/PhysRevD.101.053002

\section{INTRODUCTION}

Recently, the LHCb Collaboration has obtained the antitriplet charmed baryon lifetimes with high precision, given by [1]

$$
\begin{aligned}
& \left(\tau_{\Lambda_{c}^{+}}, \tau_{\Xi_{c}^{+}}, \tau_{\Xi_{c}^{0}}\right) \\
& \quad=(203.5 \pm 2.2,456.8 \pm 5.5,154.5 \pm 2.5) \mathrm{fs} .
\end{aligned}
$$

Note that the decay lifetime of $\Xi_{c}^{0}$ is $3 \sigma$ above the previous averaged value of $(112 \pm 12)$ fs in Particle Data Group (PDG) [2]. Furthermore, BESIII [3] and Belle [4] Collaborations have precisely measured the absolute decay branching ratio for $\Lambda_{c}^{+} \rightarrow p K^{-} \pi^{+}$with the world average of

$$
\mathcal{B}\left(\Lambda_{c}^{+} \rightarrow p K^{-} \pi^{+}\right)=(6.28 \pm 0.32) \%
$$

in PDG [2]. Moreover, Belle Collaboration has determined the absolute branching ratios in $\Xi_{c}$, given by $[5,6]$

Published by the American Physical Society under the terms of the Creative Commons Attribution 4.0 International license. Further distribution of this work must maintain attribution to the author(s) and the published article's title, journal citation, and DOI. Funded by SCOAP .

$$
\begin{aligned}
\mathcal{B}\left(\Xi_{c}^{0} \rightarrow \Xi^{-} \pi^{+}\right) & =(1.80 \pm 0.52) \%, \\
\mathcal{B}\left(\Xi_{c}^{+} \rightarrow \Xi^{-} \pi^{+} \pi^{+}\right) & =(2.86 \pm 1.27) \%,
\end{aligned}
$$

from the decay chains of $B$ mesons. These decay branching ratios are important, as most of the other branching ratios of antitriplet charmed baryons are measured relative to them.

It is known that there are some difficulties for the theoretical study in the nonleptonic decays of charmed baryons due to the failure of the factorization approach. On the other hand, one can use the $S U(3)_{F}$ flavor symmetry to relate the amplitudes among different decays [7-9]. This becomes possible [10-27] as there have been recently many new experimental measurements for charmed baryon decays [3-6, 28-37]. In addition to the analysis of charmed baryon decays with $S U(3)_{F}$, the theoretical calculations based on dynamical models have also been done in the literature [38-50]. However, the results are often not reliable and different among models. The main difficulties are due to the unknown baryon wave functions and nonfactorizable contributions.

In this work, we concentrate on the decays of $\mathbf{B}_{c} \rightarrow \mathbf{B}_{n} V$ with the $S U(3)_{F}$ flavor symmetry, where $\mathbf{B}_{c}$ and $\mathbf{B}_{n}$ correspond to the antitriplet charmed and octet baryons, and $V$ stands for the vector mesons, respectively. In fact, some of the decay branching ratios have been recently explored based on $S U(3)_{F}$ in Ref. [25]. However, the approach in Ref. [25] has ignored the contributions from color-symmetric parts of the effective Hamiltonian and 
correlations among the $S U(3)_{F}$ parameters. In addition, there should be four independent wave amplitudes [51], but only one is used in Ref. [25]. In this study, we shall include all the wave amplitudes and consider the full effective Hamiltonian. We shall also discuss the decay asymmetry parameters in $\mathbf{B}_{c} \rightarrow \mathbf{B}_{n} V$, such as the up-down and longitudinal polarization asymmetries of $\mathbf{B}_{n}$ and the asymmetry parameter of $V$.

This paper is organized as follows. In Sec. II, we present the formalism. In Sec. III, we extract the $S U(3)_{F}$ parameters from the experimental data. We conclude our study in Sec. IV.

\section{FORMALISM}

The most general form of the amplitude for $\mathbf{B}_{c} \rightarrow \mathbf{B}_{n} V$ can be written as

$$
\begin{aligned}
M= & \bar{u}_{f}\left(p_{f}\right) \epsilon^{\mu *}\left[A_{1} \gamma_{\mu} \gamma_{5}+A_{2} \frac{p_{f \mu}}{m_{i}} \gamma_{5}+B_{1} \gamma_{\mu}+B_{2} \frac{p_{f \mu}}{m_{i}}\right] \\
& \times u_{i}\left(p_{i}\right),
\end{aligned}
$$

where $\epsilon^{\mu}$ is the four-vector polarization for the vector meson of $V, u_{i}\left(p_{i}\right)$ and $u_{f}\left(p_{f}\right)$ are the four-component spinors (momenta) for the initial and final baryons, respectively, and $m_{i}$ represents the initial baryon mass. In general, the physical vector meson with its momentum in the $z$ direction has the vector polarizations of $\epsilon^{\mu}=$ $\left(0, \frac{1}{\sqrt{2}}, \frac{ \pm i}{\sqrt{2}}, 0\right)$ for $\lambda_{V}= \pm 1$ and $\epsilon^{\mu}=\left(\left|\vec{p}_{V}\right| / m_{V}, 0,0\right.$, $\left.E_{V} / m_{V}\right)$ for $\lambda_{V}=0$, where $\lambda_{V}$ is the helicity and $m_{V}$, $\vec{p}_{V}$, and $E_{V}$ are the mass, 3-momentum, and energy of the vector meson, respectively. In the center of the momentum frame (CMF), the kinematic factors of $A_{2}$ and $B_{2}$ in Eq. (4) can be further written as

$$
\epsilon^{\mu *} p_{f \mu} / m_{i}=\epsilon^{\mu *} p_{i \mu} / m_{i}=\epsilon^{0 *} .
$$

Here, we have used $p_{i}^{\mu}=p_{f}^{\mu}+p_{V}^{\mu}$ and $\epsilon_{\mu} p_{V}^{\mu}=0$, where $p_{V}$ corresponds to the 4-momentum of the vector meson. It is clear that the terms associated with $A_{2}$ and $B_{2}$ will only contribute to the decay in the case of $\lambda_{V}=0$, which are suppressed by the factor of $p_{c} / m_{V}$ with $p_{c}$ defined as the magnitude of the 3-momentum in the CMF, so that they can be ignored.

The decay width, up-down asymmetry and longitudinal polarization of $\mathbf{B}_{c} \rightarrow \mathbf{B}_{n} V$ are given by

$$
\begin{aligned}
\Gamma= & \frac{p_{c}}{4 \pi} \frac{E_{f}+m_{f}}{m_{i}} \\
& \times\left[2\left(|S|^{2}+\left|P_{2}\right|^{2}\right)+\frac{E_{V}^{2}}{m_{V}^{2}}\left(|S+D|^{2}+\left|P_{1}\right|^{2}\right)\right],
\end{aligned}
$$

$$
\begin{gathered}
\alpha=\frac{2 E_{V}^{2} \operatorname{Re}(S+D) * P_{1}+4 m_{v}^{2} \operatorname{Re}\left(S^{*} P_{2}\right)}{2 m_{V}^{2}\left(|S|^{2}+\left|P_{2}\right|^{2}\right)+E_{V}^{2}\left(|S+D|^{2}+\left|P_{1}\right|^{2}\right)}, \\
P_{L}=\frac{2 E_{V}^{2} \operatorname{Re}(S+D)^{*} P_{1}-4 m_{V}^{2} \operatorname{Re}\left(S^{*} P_{2}\right)}{2 m_{V}^{2}\left(|S|^{2}+\left|P_{2}\right|^{2}\right)+E_{V}^{2}\left(|S+D|^{2}+\left|P_{1}\right|^{2}\right)},
\end{gathered}
$$

where $S, P_{1,2}$, and $D$, corresponding to the orbital angular momenta of $l=0,1,2$ in the nonrelativistic limit, are given by [51]

$$
S=-A_{1}
$$

$$
\begin{gathered}
P_{1}=-\frac{p_{c}}{E_{V}}\left(\frac{m_{i}+m_{f}}{E_{f}+m_{f}} B_{1}+B_{2}\right), \\
P_{2}=\frac{p_{c}}{E_{f}+m_{f}} B_{1}, \\
D=-\frac{p_{c}^{2}}{E_{V}\left(E_{f}+m_{f}\right)}\left(A_{1}-A_{2}\right),
\end{gathered}
$$

respectively. Here, $\alpha$ and $P_{L}$ are defined by

$$
\begin{gathered}
\frac{d \Gamma}{d \cos \theta} \propto 1+\alpha \cos \theta, \\
P_{L}=\frac{\Gamma\left(\lambda_{\mathbf{B}_{n}}=1\right)-\Gamma\left(\lambda_{\mathbf{B}_{n}}=-1\right)}{\Gamma\left(\lambda_{\mathbf{B}_{n}}=1\right)+\Gamma\left(\lambda_{\mathbf{B}_{n}}=-1\right)},
\end{gathered}
$$

where $d \Gamma$ is the partial decay width, $\lambda_{\mathbf{B}_{n}}$ is the helicity of $\mathbf{B}_{n}$, and $\theta$ is the angle between the spin and momentum directions of $\mathbf{B}_{c}$ and $\mathbf{B}_{n}$, respectively.

Since the vector meson of $V$ subsequently decays into two pseudoscalar mesons, its polarization can be determined. As a result, we can discuss the decay asymmetry parameter of $V$, defined by [38]

$$
\frac{d \Gamma_{V}}{d \cos \theta_{V}} \propto 1+\alpha_{V} \cos ^{2} \theta_{V}
$$

with

$$
\alpha_{V}=\frac{E_{V}^{2}\left(|S+D|^{2}+\left|P_{1}\right|^{2}\right)-m_{V}^{2}\left(|S|^{2}+\left|P_{2}\right|^{2}\right)}{m_{V}^{2}\left(|S|^{2}+\left|P_{2}\right|^{2}\right)},
$$

where $d \Gamma_{V}$ is the partial decay width for the $V$ decay and $\theta_{V}$ is the polar angle between $\vec{p}_{V}$ and the momentum directions of the pseudoscalar mesons in the CMF of $V$.

The effective Hamiltonian responsible for the decay processes with $\Delta c=-1$ is 


$$
\begin{aligned}
& \mathcal{H}_{\mathrm{eff}} \\
& =\frac{G_{F}}{\sqrt{2}} \sum_{q, q^{\prime}=d, s}\left[c_{1} V_{u q}^{*} V_{c q^{\prime}}(\bar{u} q)\left(\overline{q^{\prime}} c\right)+c_{2} V_{u q}^{*} V_{c q^{\prime}}(\bar{u} q)\left(\bar{q}^{\prime} c\right)\right],
\end{aligned}
$$

where the quark operators are defined as $\left(\bar{q}_{1} q_{2}\right)=$ $\left(\bar{q}_{1} \gamma_{\mu}\left(1-\gamma_{5}\right) q_{2}\right)$ with summing over the colors, the Wilson coefficient of $c_{1}\left(c_{2}\right)$ is $1.246(-0.636)$ at the scale of $\mu=1.25 \mathrm{GeV}$ [52], and $G_{F}$ is the Fermi constant. Note that $\left(q, q^{\prime}\right)=(d, s),(d, d)$, or $(s, s)$ and $(s, d)$ correspond to the Cabibbo allowed, singly Cabibbo suppressed, and doubly Cabibbo suppressed decays, respectively.
By using the Cabibbo-Kobayashi-Maskawa mixing parameters of $V_{c s}=V_{u d} \approx 1$ and $s_{c} \equiv V_{u s}=-V_{c d} \approx$ 0.225 , the effective Hamiltonian in the flavor basis is given by

$\mathcal{H}_{\mathrm{eff}}=\frac{G_{F}}{2 \sqrt{2}}\left(\frac{c_{-}}{2} \epsilon^{l i j} H(6)_{l k}+c_{+} H(\overline{15})_{k}^{i j}\right)\left(\bar{q}_{j} q^{k}\right)\left(\bar{q}_{i} c\right)$,

where $\left(q_{1}, q_{2}, q_{3}\right)=(u, d, s), c_{ \pm}=c_{1} \pm c_{2}$, and $\epsilon^{l i j}$ represents the total antisymmetric tensor with $\epsilon^{123}=1$. Here, the tensor components are given by

$$
\begin{aligned}
H(6)_{i j} & =\left(\begin{array}{ccc}
0 & 0 & 0 \\
0 & 2 & 2 s_{c} \\
0 & 2 s_{c} & 2 s_{c}^{2}
\end{array}\right), \\
H\left(\overline{15}_{k}^{i j}\right. & =\left(\left(\begin{array}{lll}
0 & 0 & 0 \\
0 & 0 & 0 \\
0 & 0 & 0
\end{array}\right),\left(\begin{array}{ccc}
0 & -s_{c} & 1 \\
-s_{c} & 0 & 0 \\
1 & 0 & 0
\end{array}\right),\left(\begin{array}{ccc}
0 & -s_{c}^{2} & s_{c} \\
-s_{c}^{2} & 0 & 0 \\
s_{c} & 0 & 0
\end{array}\right)\right) .
\end{aligned}
$$

Two of the creation operators generated by $H(\overline{15})$ are symmetric in color. As a result, $H(\overline{15})$ does not contribute to the nonfactorizable amplitudes since the charmed baryons are total antisymmetric in color [53,54].

We separate $A_{1}$ and $B_{1}$ into 6 and $\overline{15}$ parts under the $S U(3)_{F}$ symmetry,

$$
\begin{aligned}
& A_{1}=A_{1}^{(6)}+A_{1}^{(\overline{15})}, \\
& B_{1}=B_{1}^{(6)}+B_{1}^{(\overline{15})} .
\end{aligned}
$$

In Eq. (20), $A_{1}^{(6)}$ and $B_{1}^{(6)}$ are parametrized as

$$
\begin{aligned}
A_{1}^{(6)}\left(\mathbf{B}_{c} \rightarrow \mathbf{B}_{n} V\right)= & a_{0} H(6)_{i j}\left(\mathbf{B}_{c}^{\prime}\right)^{i k}\left(\mathbf{B}_{n}\right)_{k}^{j}(V)_{l}^{l}+a_{1} H_{i j}(6)\left(\mathbf{B}_{c}^{\prime}\right)^{i k}\left(\mathbf{B}_{n}\right)_{k}^{l}(V)_{l}^{j} \\
& +a_{2} H_{i j}(6)\left(\mathbf{B}_{c}^{\prime}\right)^{i k}(V)_{k}^{l}\left(\mathbf{B}_{n}\right)_{l}^{j}+a_{3} H_{i j}(6)\left(\mathbf{B}_{n}\right)_{k}^{i}(V)_{l}^{j}\left(\mathbf{B}_{c}^{\prime}\right)^{k l}, \\
B_{1}^{(6)}\left(\mathbf{B}_{c} \rightarrow \mathbf{B}_{n} V\right)= & b_{0} H(6)_{i j}\left(\mathbf{B}_{c}^{\prime}\right)^{i k}\left(\mathbf{B}_{n}\right)_{k}^{j}(V)_{l}^{l}+b_{1} H_{i j}(6)\left(\mathbf{B}_{c}^{\prime}\right)^{i k}\left(\mathbf{B}_{n}\right)_{k}^{l}(V)_{l}^{j} \\
& +b_{2} H_{i j}(6)\left(\mathbf{B}_{c}^{\prime}\right)^{i k}(V)_{k}^{l}\left(\mathbf{B}_{n}\right)_{l}^{j}+b_{3} H_{i j}(6)\left(\mathbf{B}_{n}\right)_{k}^{i}(V)_{l}^{j}\left(\mathbf{B}_{c}^{\prime}\right)^{k l},
\end{aligned}
$$

where $a_{i}$ and $b_{i}$ are the $S U(3)_{F}$ parameters, while $\mathbf{B}_{c, n}$ and $V$ can be written under the tensor components of the $S U(3)_{F}$ representations, given by

$$
\begin{aligned}
\mathbf{B}_{c} & =\left(\Xi_{c}^{0},-\Xi_{c}^{+}, \Lambda_{c}^{+}\right), \\
\mathbf{B}_{n} & =\left(\begin{array}{ccc}
\frac{1}{\sqrt{6}} \Lambda+\frac{1}{\sqrt{2}} \Sigma^{0} & \Sigma^{+} & p \\
\Sigma^{-} & \frac{1}{\sqrt{6}} \Lambda-\frac{1}{\sqrt{2}} \Sigma^{0} & n \\
\Xi^{-} & \Xi^{0} & -\sqrt{\frac{2}{3}} \Lambda
\end{array}\right),
\end{aligned}
$$


and

$$
V=\left(\begin{array}{ccc}
\frac{1}{\sqrt{2}}\left(\omega+\rho^{0}\right) & \rho^{+} & K^{*+} \\
\rho^{-} & \frac{1}{\sqrt{2}}\left(\omega-\rho^{0}\right) & K^{* 0} \\
K^{*-} & \bar{K}^{* 0} & \phi
\end{array}\right),
$$

respectively.

On the other hand, the contribution from $H(\overline{15})$ to $c \rightarrow$ $u q^{\prime} \bar{q}$ is factorizable, given by

$$
\begin{aligned}
M(\overline{15})= & \frac{G_{F}}{2 \sqrt{2}} V_{u q} V_{c q^{\prime}} c_{+}\left(1+\frac{1}{N_{c}}\right) \\
& \times\langle V|(\bar{u} q)| 0\rangle\left\langle\mathbf{B}_{n}\left|\left(\bar{q}^{\prime} c\right)\right| \mathbf{B}_{c}\right\rangle
\end{aligned}
$$

for the vector mesons with positive charges, while the creation operators $\bar{q}^{\prime}$ and $\bar{u}$ are interchanged for the neutral vector mesons. Accordingly, $A_{1}^{(\overline{15})}$ and $B_{1}^{(\overline{15})}$ in Eq. (20) are given by [50]

$$
\begin{aligned}
A_{1}^{(\overline{15})}= & -\frac{G_{F}}{2 \sqrt{2}} V_{u q} V_{c q^{\prime}} f_{V} m_{V} c_{+} \\
& \times\left(1+\frac{1}{N_{c}}\right)\left(g_{1}-g_{2} \frac{m_{i}-m_{f}}{m_{i}}\right), \\
B_{1}^{(\overline{15})}= & \frac{G_{F}}{2 \sqrt{2}} V_{u q} V_{c q^{\prime}} f_{V} m_{V} c_{+} \\
& \times\left(1+\frac{1}{N_{c}}\right)\left(f_{1}+f_{2} \frac{m_{i}+m_{f}}{m_{i}}\right),
\end{aligned}
$$

where $\left\langle V\left|\left(\bar{q} q^{\prime}\right)\right| 0\right\rangle=f_{V} m_{V} \epsilon_{\mu}^{*}$ and $N_{c}$ is the effective color number. In Eq. (26), we take that $f_{V}=0.215 \mathrm{GeV}$ and the form factors of $f_{i}\left(g_{i}\right)$ are defined by

$$
\begin{aligned}
\left\langle\mathbf{B}_{n}|(\bar{q} c)| \mathbf{B}_{c}\right\rangle & =\bar{u}_{f}\left(p_{f}\right)\left[\left(f_{1} \gamma_{\mu}-f_{2} i \sigma_{\mu \nu} \frac{q^{\nu}}{m_{i}}+f_{3} \frac{q_{\mu}}{m_{i}}\right)\right. \\
& \left.-\left(g_{1} \gamma_{\mu}-g_{2} i \sigma_{\mu \nu} \frac{q^{\nu}}{m_{i}}+g_{3} \frac{q_{\mu}}{m_{i}}\right) \gamma_{5}\right] u_{i}\left(p_{i}\right) .
\end{aligned}
$$

In our calculation, we evaluate the form factors from the MIT bag model $[55,56]$. The baryon wave functions and form factors are listed in the Appendix A.

The factorizable parts in $A_{2}$ and $B_{2}$ are given by

$$
\begin{aligned}
A_{2}^{(\mathrm{fac})}= & \frac{G_{F}}{\sqrt{2}}\left[c_{+}\left(1+\frac{1}{N_{c}}\right) \pm c_{-}\left(1-\frac{1}{N_{c}}\right)\right] \\
& \times V_{u q} V_{c q^{\prime}} f_{V} m_{V} g_{2},
\end{aligned}
$$

$$
\begin{aligned}
B_{2}^{(\mathrm{fac})}= & -\frac{G_{F}}{\sqrt{2}}\left[c_{+}\left(1+\frac{1}{N_{c}}\right) \pm c_{-}\left(1-\frac{1}{N_{c}}\right)\right] \\
& \times V_{u q} V_{c q^{\prime}} f_{V} m_{V} f_{2},
\end{aligned}
$$

with the " \pm " signs for mesons with positive and neutral charges, respectively. In general, it is also possible to parametrize the nonfactorizable contributions in $A_{2}$ and $B_{2}$ according to the $S U(3)_{F}$ symmetry. However, since they are suppressed due to Eq. (5), we will neglect these parts.

To sum up, $A_{1}^{(\overline{15})}\left(B_{1}^{(\overline{15})}\right)$ and $A_{2}^{(\mathrm{fac})}\left(B_{2}^{(\mathrm{fac})}\right)$ can be calculated from the factorization approach, while $A_{1}^{(6)}\left(B_{1}^{(6)}\right)$ are parametrized by the $S U(3)_{F}$ symmetry. The detailed results are shown in Appendix B.

\section{NUMERICAL RESULTS}

The effective color number can be extracted from the decay branching ratio of $\Lambda_{c}^{+} \rightarrow p \phi$ since it only receives the factorizable contribution $[43,53]$. The decay amplitude is given by

$$
\begin{aligned}
M\left(\Lambda_{c}^{+} \rightarrow p \phi\right)= & \frac{G_{F}}{\sqrt{2}} V_{u s} V_{c s}\left(c_{2}+\frac{c_{1}}{N_{c}}\right) \\
& \times\langle\phi|(\bar{s} s)| 0\rangle\left\langle p|(\bar{u} c)| \Lambda_{c}\right\rangle
\end{aligned}
$$

With the form factors given in Appendix A, we obtain the decay parameters

$$
\begin{aligned}
\alpha\left(\Lambda_{c}^{+} \rightarrow p \phi\right) & =-0.08, \\
P_{L}\left(\Lambda_{c}^{+} \rightarrow p \phi\right) & =-0.85, \quad \alpha_{V}\left(\Lambda_{c}^{+} \rightarrow p \phi\right)=0.97,
\end{aligned}
$$

which are independent of $N_{c}$. On the other hand, with the experimental data of $\mathcal{B}\left(\Lambda_{c}^{+} \rightarrow p \phi\right)=(1.06 \pm 0.14) \times$ $10^{-3}$ [2], we find that $\left(c_{2}+c_{1} / N_{c}\right)=0.49$, leading to $N_{c}=9$, while the effective coupling strengths are found to be

$$
\begin{aligned}
& A_{1}\left(\Lambda_{c}^{+} \rightarrow p \phi\right)=0.0110 G_{F} \mathrm{GeV}^{2} \\
& B_{1}\left(\Lambda_{c}^{+} \rightarrow p \phi\right)=-0.0175 G_{F} \mathrm{GeV}^{2} \\
& A_{2}\left(\Lambda_{c}^{+} \rightarrow p \phi\right)=0.0034 G_{F} \mathrm{GeV}^{2} \\
& B_{2}\left(\Lambda_{c}^{+} \rightarrow p \phi\right)=0.0109 G_{F} \mathrm{GeV}^{2}
\end{aligned}
$$

For the other decay modes of $\mathbf{B}_{c} \rightarrow B_{n} V$, the nonfactorizable effects in $A_{1}^{(6)}$ and $B_{1}^{(6)}$ are sizable, which cannot be ignored. The calculations of the nonfactorizable amplitudes are nonperturbative, which are generally model dependent. To tackle these effects, we determine the parameters in Eqs. (21) and (22) with the experimental data, which are listed in Table I. Here, we have used 
TABLE I. Decay branching ratios of $\mathbf{B}_{c} \rightarrow \mathbf{B}_{n} V$ from the experimental data and our $S U(3)_{F}$ reconstructed values.

\begin{tabular}{|c|c|c|c|c|c|}
\hline Channel & $10^{2} \mathcal{B}_{\mathrm{ex}}$ & $10^{2} \mathcal{B}_{S U(3)}$ & Channel & $10^{3} \mathcal{B}_{\mathrm{ex}}$ & $10^{3} \mathcal{B}_{S U(3)}$ \\
\hline$\Lambda_{c}^{+} \rightarrow \Lambda^{0} \rho^{+}$ & $<6[2,59]$ & $4.81 \pm 0.58$ & $\Lambda_{c}^{+} \rightarrow p \omega$ & $0.94 \pm 0.39[2]$ & $0.63 \pm 0.34$ \\
\hline$\Lambda_{c}^{+} \rightarrow \Sigma^{+} \omega$ & $1.70 \pm 0.21$ & $1.81 \pm 0.19$ & $\Lambda_{c}^{+} \rightarrow \Sigma^{+} K^{* 0}$ & $3.5 \pm 1.0[2]$ & $0.38 \pm 0.09$ \\
\hline$\Lambda_{c}^{+} \rightarrow p \bar{K}^{* 0}$ & $1.96 \pm 0.27[2]$ & $2.03 \pm 0.25$ & $\Lambda_{c}^{+} \rightarrow p \phi$ & $1.06 \pm 0.14[2]$ & $0.87 \pm 0.14$ \\
\hline$\Lambda_{c}^{+} \rightarrow \Sigma^{+} \phi$ & $0.39 \pm 0.06[2]$ & $0.39 \pm 0.06$ & $\Xi_{c}^{+} \rightarrow p \bar{K}^{* 0}$ & $4.13 \pm 1.69[2,6]$ & $4.71 \pm 1.22$ \\
\hline$\Lambda_{c}^{+} \rightarrow \Sigma^{+} \rho^{0}$ & $1.0 \pm 0.5[2]$ & $1.43 \pm 0.42$ & $\Xi_{c}^{+} \rightarrow \Sigma^{+} \phi$ & $1.17 \pm 0.87[2,6,57]$ & $1.82 \pm 0.40$ \\
\hline$\Xi_{c}^{+} \rightarrow \Sigma^{+} \bar{K}^{* 0}$ & $2.88 \pm 1.06[2,6]$ & $1.40 \pm 0.69$ & $\Xi_{c}^{0} \rightarrow \Lambda^{0} \phi$ & $0.49 \pm 0.15[2]$ & $0.44 \pm 0.08$ \\
\hline$\Xi_{c}^{+} \rightarrow \Xi^{0} \rho^{+}$ & $8.2 \pm 3.6[2,6]$ & $14.48 \pm 2.44$ & & & \\
\hline
\end{tabular}

Eq. (C14) in Appendix $C$ to exact the branching ratios in $\Xi_{c}^{+}$. In particular, we have $\mathcal{B}\left(\Xi_{c}^{+} \rightarrow \Xi^{0} \rho^{+}\right) \approx \mathcal{B}\left(\Xi_{c}^{+} \rightarrow\right.$ $\left.\Xi^{0} \pi^{+} \pi^{0}\right)=(8.3 \pm 3.6) \%$ as the experimental branching ratio, as stated in Appendix C. In addition, the branching ratios of $\Xi_{c}^{+} \rightarrow \Sigma^{+} \phi$ and $\Lambda_{c}^{+} \rightarrow \Sigma^{+} \rho^{0}$ can be obtained by the event counting method in Refs. [57,58]. For $\Lambda_{c}^{+} \rightarrow p \phi$, we impose $10 \%$ error deviations for the effective coupling strengths in Eq. (32) to account for the errors in the form factors evaluated from the MIT bag model.

In our numerical calculations, we adopt the minimal $\chi^{2}$ fitting. We find that the minimal value of $\chi^{2} /$ (degree of freedom) is given by $18 / 4=4.5$, which is reasonable as $S U(3)_{F}$ is not an exact symmetry. The results of the effective coupling parameters are found to be

$$
\begin{gathered}
\left(a_{1}, a_{2}, a_{3}, \tilde{a}\right)=(-2.40 \pm 0.24,0.82 \pm 0.44,-2.05 \pm 0.38,-1.59 \pm 0.10) G_{F} \mathrm{GeV}^{2} \\
\left(b_{1}, b_{2}, b_{3}, \tilde{b}\right)=(6.91 \pm 0.28,-0.82 \pm 0.99,2.82 \pm 0.52,0.75 \pm 0.42) G_{F} \mathrm{GeV}^{2}
\end{gathered}
$$

with the correlation in the sequences $\left(a_{1}, a_{2}, a_{3}, \tilde{a}, b_{1}, b_{2}, b_{3}, \tilde{b}\right)$ given by

$$
R=\left(\begin{array}{cccccccc}
1 & -0.087 & 0.085 & -0.043 & 0.423 & 0.161 & -0.091 & 0.083 \\
-0.087 & 1 & 0.599 & 0.325 & 0.043 & 0.540 & 0.105 & 0.363 \\
0.085 & 0.599 & 1 & -0.094 & 0.126 & 0.257 & 0.346 & -0.096 \\
-0.043 & 0.325 & -0.094 & 1 & -0.011 & 0.473 & -0.308 & 0.640 \\
0.423 & 0.043 & 0.126 & -0.011 & 1 & 0.314 & 0.135 & -0.150 \\
0.161 & 0.540 & 0.257 & 0.473 & 0.314 & 1 & -0.112 & 0.472 \\
-0.091 & 0.105 & 0.346 & -0.308 & 0.135 & -0.112 & 1 & -0.355 \\
0.083 & 0.363 & -0.096 & 0.640 & -0.150 & 0.472 & -0.355 & 1
\end{array}\right),
$$

where $\tilde{a}=a_{0}+\left(a_{1}+a_{2}-a_{3}\right) / 3$ and $\tilde{b}=b_{0}+\left(b_{1}+\right.$ $\left.b_{2}-b_{3}\right) / 3$. Accordingly, the branching ratios, up-down asymmetries, and longitudinal polarizations for the Cabibbo allowed, singly suppressed, and doubly suppressed decays of $\mathbf{B}_{c} \rightarrow \mathbf{B}_{n} V$ are shown in Tables II-IV, respectively. The reconstructed branching ratios are also listed in Table I. Most of the results are consistent with the experimental data except $\mathcal{B}\left(\Lambda_{c}^{+} \rightarrow \Sigma^{+} K^{* 0}\right)$. The errors in our results arise from the uncertainties in the $S U(3)_{F}$ parameters, which could be viewed as error propagations from the data. With more experimental data in the future, the $S U(3)_{F}$ breaking effects could also be systematically considered with additional parameters $[8,15,27]$. In this work, we merely note that our results might have around $20 \%$ deviations caused by the $S U(3)_{F}$ breaking.

Note that our result of $\mathcal{B}\left(\Lambda_{c}^{+} \rightarrow \Lambda^{0} \rho^{+}\right)=(4.81 \pm$ $0.58) \times 10^{-2}$ agrees with the experimental upper limit of $6 \times 10^{-2}$ (90\% C.L.) [2,59], which is obtained from $\mathcal{B}\left(\Lambda_{c}^{+} \rightarrow \Lambda^{0} \pi^{+} \pi^{0}\right) / \mathcal{B}\left(\Lambda_{c}^{+} \rightarrow p K^{-} \pi^{+}\right)<0.95$ by CLEO [59], where the resonant contribution of $\mathcal{B}\left(\Lambda_{c}^{+} \rightarrow \Lambda^{0} \rho^{+}\right.$, $\left.\rho^{+} \rightarrow \pi^{+} \pi^{0}\right)$ is included in $\mathcal{B}\left(\Lambda_{c}^{+} \rightarrow \Lambda^{0} \pi^{+} \pi^{0}\right)$ along with $\mathcal{B}\left(\Lambda_{c}^{+} \rightarrow p K^{-} \pi^{+}\right)=(6.28 \pm 0.32) \%$. However, it is inconsistent with the latest experimental measurement of $\mathcal{B}\left(\Lambda_{c}^{+} \rightarrow\right.$ $\left.\Lambda^{0} \pi^{+} \pi^{0}\right) / \mathcal{B}\left(\Lambda_{c}^{+} \rightarrow p K^{-} \pi^{+}\right)=1.20 \pm 0.11$ [3], making the experimental upper limit for $\mathcal{B}\left(\Lambda_{c}^{+} \rightarrow \Lambda^{0} \rho^{+}\right)$questionable.

The other possible resonant dominated contribution in $\Lambda_{c}^{+} \rightarrow \Lambda^{0} \pi^{+} \pi^{0}$ is given by 
TABLE II. Cabibbo allowed decays of $\mathbf{B}_{c} \rightarrow \mathbf{B}_{n} V$.

\begin{tabular}{|c|c|c|c|c|}
\hline Channel & $10^{2} \mathcal{B}_{S U(3)}$ & $\alpha$ & $P_{L}$ & $\alpha_{V}$ \\
\hline$\Lambda_{c}^{+} \rightarrow \Lambda^{0} \rho^{+}$ & $4.81 \pm 0.58^{\mathrm{a}}$ & $-0.27 \pm 0.04$ & $-0.93 \pm 0.05$ & $2.01 \pm 0.39$ \\
\hline$\Lambda_{c}^{+} \rightarrow p \bar{K}^{* 0}$ & $2.03 \pm 0.25^{\mathrm{a}}$ & $-0.18 \pm 0.05$ & $-0.62 \pm 0.16$ & $4.96 \pm 0.76$ \\
\hline$\Lambda_{c}^{+} \rightarrow \Sigma^{0} \rho^{+}$ & $1.43 \pm 0.42$ & $-0.34 \pm 0.18$ & $-0.66 \pm 0.34$ & $9.82 \pm 7.19$ \\
\hline$\Lambda_{c}^{+} \rightarrow \Sigma^{+} \rho^{0}$ & $1.43 \pm 0.42^{\mathrm{a}}$ & $-0.34 \pm 0.18$ & $-0.66 \pm 0.34$ & $9.82 \pm 7.19$ \\
\hline$\Lambda_{c}^{+} \rightarrow \Sigma^{+} \omega$ & $1.81 \pm 0.19^{\mathrm{a}}$ & $-0.34 \pm 0.11$ & $-0.67 \pm 0.22$ & $1.60 \pm 0.62$ \\
\hline$\Lambda_{c}^{+} \rightarrow \Sigma^{+} \phi$ & $0.39 \pm 0.06^{\mathrm{a}}$ & $0.02 \pm 0.03$ & $0.08 \pm 0.10$ & $0.16 \pm 0.01$ \\
\hline$\Lambda_{c}^{+} \rightarrow \Xi^{0} K^{*+}$ & $0.10 \pm 0.10$ & $-0.15 \pm 0.20$ & $-0.40 \pm 0.55$ & $0.35 \pm 0.52$ \\
\hline$\Xi_{c}^{+} \rightarrow \Sigma^{+} \bar{K}^{* 0}$ & $1.40 \pm 0.69^{\mathrm{a}}$ & $0.32 \pm 0.30$ & $0.37 \pm 0.35$ & $40.30_{-41.30}^{+68.54}$ \\
\hline$\Xi_{c}^{+} \rightarrow \Xi^{0} \rho^{+}$ & $14.48 \pm 2.44^{\mathrm{a}}$ & $0.00 \pm 0.07$ & $-0.62 \pm 0.13$ & $1.07 \pm 0.09$ \\
\hline$\Xi_{c}^{0} \rightarrow \Lambda^{0} \bar{K}^{* 0}$ & $1.37 \pm 0.26$ & $-0.28 \pm 0.10$ & $-0.67 \pm 0.24$ & $6.94 \pm 2.28$ \\
\hline$\Xi_{c}^{0} \rightarrow \Sigma^{0} \bar{K}^{* 0}$ & $0.42 \pm 0.23$ & $-0.33 \pm 0.50$ & $-0.42 \pm 0.62$ & $38.99_{-39.99}^{+82.32}$ \\
\hline$\Xi_{c}^{0} \rightarrow \Sigma^{+} K^{*-}$ & $0.24 \pm 0.17$ & $-0.37 \pm 0.31$ & $-0.76_{-0.24}^{+0.64}$ & $1.94 \pm 2.63$ \\
\hline$\Xi_{c}^{0} \rightarrow \Xi^{0} \rho^{0}$ & $0.88 \pm 0.22$ & $-0.15 \pm 0.18$ & $-0.26 \pm 0.32$ & $20.55 \pm 5.91$ \\
\hline$\Xi_{c}^{0} \rightarrow \Xi^{0} \omega$ & $2.78 \pm 0.45$ & $-0.40 \pm 0.07$ & $-0.71 \pm 0.12$ & $2.03 \pm 0.47$ \\
\hline$\Xi_{c}^{0} \rightarrow \Xi^{0} \phi$ & $0.14 \pm 0.13$ & $0.22 \pm 0.10$ & $0.61 \pm 0.27$ & $0.71 \pm 0.51$ \\
\hline$\Xi_{c}^{0} \rightarrow \Xi^{-} \rho^{+}$ & $8.98 \pm 0.55$ & $-0.32 \pm 0.01$ & $-0.94 \pm 0.01$ & $2.45 \pm 0.21$ \\
\hline
\end{tabular}

${ }^{\mathrm{a}}$ Reconstructed values.

TABLE III. Singly Cabibbo suppressed decays of $\mathbf{B}_{c} \rightarrow \mathbf{B}_{n} V$.

\begin{tabular}{|c|c|c|c|c|}
\hline Channel & $10^{3} \mathcal{B}_{S U(3)}$ & $\alpha$ & $P_{L}$ & $\alpha_{V}$ \\
\hline$\Lambda_{c}^{+} \rightarrow \Lambda^{0} K^{*+}$ & $3.35 \pm 0.37$ & $-0.13 \pm 0.05$ & $-0.81 \pm 0.09$ & $1.03 \pm 0.22$ \\
\hline$\Lambda_{c}^{+} \rightarrow p \rho^{0}$ & $0.02_{-0.02}^{+0.07}$ & $-0.27_{-0.73}^{+1.27}$ & $-0.28_{-0.72}^{+1.28}$ & $\ldots$ \\
\hline$\Lambda_{c}^{+} \rightarrow p \omega$ & $0.63 \pm 0.34^{\mathrm{a}}$ & $0.36 \pm 0.17$ & $0.88_{-0.21}^{+0.12}$ & $2.95 \pm 1.01$ \\
\hline$\Lambda_{c}^{+} \rightarrow p \phi$ & $0.87 \pm 0.14^{\mathrm{a}}$ & $-0.06 \pm 0.04$ & $-0.83 \pm 0.08$ & $0.88 \pm 0.14$ \\
\hline$\Lambda_{c}^{+} \rightarrow n \rho^{+}$ & $1.76 \pm 0.72$ & $-0.09 \pm 0.22$ & $-0.84_{-0.16}^{+0.35}$ & $1.48 \pm 0.47$ \\
\hline$\Lambda_{c}^{+} \rightarrow \Sigma^{0} K^{*+}$ & $0.18 \pm 0.04$ & $-0.14 \pm 0.17$ & $-0.35 \pm 0.41$ & $11.71 \pm 5.00$ \\
\hline$\Lambda_{c}^{+} \rightarrow \Sigma^{+} K^{* 0}$ & $0.38 \pm 0.09^{\mathrm{a}}$ & $-0.14 \pm 0.17$ & $-0.34 \pm 0.41$ & $11.86 \pm 4.99$ \\
\hline$\Xi_{c}^{+} \rightarrow \Lambda^{0} \rho^{+}$ & $1.52 \pm 0.57$ & $0.49 \pm 0.22$ & $0.28 \pm 0.46$ & $2.05 \pm 0.82$ \\
\hline$\Xi_{c}^{+} \rightarrow p \bar{K}^{* 0}$ & $4.71 \pm 1.22^{\mathrm{a}}$ & $-0.12 \pm 0.15$ & $-0.23 \pm 0.29$ & $13.01 \pm 1.39$ \\
\hline$\Xi_{c}^{+} \rightarrow \Sigma^{0} \rho^{+}$ & $11.45 \pm 1.52$ & $-0.39 \pm 0.02$ & $-0.96 \pm 0.00$ & $3.32 \pm 0.67$ \\
\hline$\Xi_{c}^{+} \rightarrow \Sigma^{+} \rho^{0}$ & $2.85 \pm 0.81$ & $-0.42 \pm 0.04$ & $-0.91_{-0.09}^{+0.12}$ & $4.99 \pm 2.14$ \\
\hline$\Xi_{c}^{+} \rightarrow \Sigma^{+} \omega$ & $4.11 \pm 0.77$ & $-0.13 \pm 0.17$ & $-0.48 \pm 0.28$ & $1.68 \pm 0.23$ \\
\hline$\Xi_{c}^{+} \rightarrow \Sigma^{+} \phi$ & $1.82 \pm 0.40^{\mathrm{a}}$ & $-0.56 \pm 0.02$ & $-0.75 \pm 0.06$ & $3.19 \pm 1.56$ \\
\hline$\Xi_{c}^{+} \rightarrow \Xi^{0} K^{*+}$ & $4.28 \pm 1.64$ & $0.28 \pm 0.10$ & $-0.45 \pm 0.27$ & $0.40 \pm 0.07$ \\
\hline$\Xi_{c}^{0} \rightarrow \Lambda^{0} \rho^{0}$ & $0.13 \pm 0.11$ & $0.51 \pm 0.17$ & $0.72 \pm 0.21$ & $13.22 \pm 7.92$ \\
\hline$\Xi_{c}^{0} \rightarrow \Lambda^{0} \omega$ & $1.51 \pm 0.20$ & $-0.16 \pm 0.19$ & $-0.19 \pm 0.31$ & $2.12 \pm 0.19$ \\
\hline$\Xi_{c}^{0} \rightarrow \Lambda^{0} \phi$ & $0.44 \pm 0.08^{\mathrm{a}}$ & $-0.10 \pm 0.13$ & $-0.63 \pm 0.32$ & $0.90 \pm 0.36$ \\
\hline$\Xi_{c}^{0} \rightarrow p K^{*-}$ & $0.19 \pm 0.14$ & $-0.47 \pm 0.26$ & $-0.88_{-0.12}^{+0.49}$ & $3.36 \pm 3.92$ \\
\hline$\Xi_{c}^{0} \rightarrow n \bar{K}^{* 0}$ & $2.52 \pm 0.79$ & $-0.31 \pm 0.19$ & $-0.58 \pm 0.36$ & $10.29 \pm 3.73$ \\
\hline$\Xi_{c}^{0} \rightarrow \Sigma^{0} \rho^{0}$ & $0.11 \pm 0.10$ & $-0.08 \pm 0.25$ & $-0.28 \pm 0.69$ & $6.42 \pm 4.14$ \\
\hline$\Xi_{c}^{0} \rightarrow \Sigma^{0} \omega$ & $0.70 \pm 0.13$ & $-0.13 \pm 0.17$ & $-0.48 \pm 0.28$ & $1.70 \pm 0.24$ \\
\hline$\Xi_{c}^{0} \rightarrow \Sigma^{0} \phi$ & $0.30 \pm 0.07$ & $-0.57 \pm 0.02$ & $-0.75 \pm 0.06$ & $3.21 \pm 1.57$ \\
\hline$\Xi_{c}^{0} \rightarrow \Sigma^{+} \rho^{-}$ & $0.19 \pm 0.13$ & $-0.50 \pm 0.32$ & $-0.83_{-0.17}^{+0.53}$ & $3.24 \pm 4.27$ \\
\hline$\Xi_{c}^{0} \rightarrow \Sigma^{-} \rho^{+}$ & $5.56 \pm 0.34$ & $-0.37 \pm 0.01$ & $-0.97 \pm 0.01$ & $3.32 \pm 0.27$ \\
\hline$\Xi_{c}^{0} \rightarrow \Xi^{0} K^{* 0}$ & $0.79 \pm 0.23$ & $-0.33 \pm 0.15$ & $-0.71_{-0.29}^{+0.32}$ & $7.39 \pm 6.19$ \\
\hline$\Xi_{c}^{0} \rightarrow \Xi^{-} K^{*+}$ & $3.36 \pm 0.23$ & $-0.12 \pm 0.01$ & $-0.87 \pm 0.03$ & $1.07 \pm 0.09$ \\
\hline
\end{tabular}

\footnotetext{
${ }^{\mathrm{a}}$ Reconstructed values.
} 
TABLE IV. Doubly Cabibbo suppressed decays of $\mathbf{B}_{c} \rightarrow \mathbf{B}_{n} V$.

\begin{tabular}{|c|c|c|c|c|}
\hline Channel & $10^{4} \mathcal{B}_{S U(3)}$ & $\alpha$ & $P_{L}$ & $\alpha_{V}$ \\
\hline$\Lambda_{c}^{+} \rightarrow p K^{* 0}$ & $0.04_{-0.04}^{+0.05}$ & $0.83_{-0.21}^{+0.17}$ & $0.38 \pm 0.55$ & $2.13_{-3.13}^{+3.35}$ \\
\hline$\Lambda_{c}^{+} \rightarrow n K^{*+}$ & $1.48 \pm 0.25$ & $-0.01 \pm 0.06$ & $-0.78 \pm 0.12$ & $0.99 \pm 0.11$ \\
\hline$\Xi_{c}^{+} \rightarrow \Lambda^{0} K^{*+}$ & $0.34_{-0.34}^{+0.37}$ & $0.59 \pm 0.27$ & $0.62 \pm 0.31$ & $10.37_{-11.37}^{+14.78}$ \\
\hline$\Xi_{c}^{+} \rightarrow p \rho^{0}$ & $0.22 \pm 0.17$ & $-0.58 \pm 0.25$ & $-0.92_{-0.08}^{+0.39}$ & $5.07 \pm 5.72$ \\
\hline$\Xi_{c}^{+} \rightarrow p \omega$ & $1.66 \pm 0.70$ & $0.42 \pm 0.17$ & $0.69 \pm 0.28$ & $3.55 \pm 0.81$ \\
\hline$\Xi_{c}^{+} \rightarrow p \phi$ & $2.29 \pm 0.39$ & $-0.40 \pm 0.01$ & $-0.93 \pm 0.03$ & $3.76 \pm 1.04$ \\
\hline$\Xi_{c}^{+} \rightarrow n \rho^{+}$ & $0.43 \pm 0.33$ & $-0.58 \pm 0.25$ & $-0.92_{-0.08}^{+0.39}$ & $5.06 \pm 5.72$ \\
\hline$\Xi_{c}^{+} \rightarrow \Sigma^{0} K^{*+}$ & $3.08 \pm 0.20$ & $-0.16 \pm 0.01$ & $-0.96 \pm 0.02$ & $1.56 \pm 0.12$ \\
\hline$\Xi_{c}^{+} \rightarrow \Sigma^{+} K^{* 0}$ & $0.40 \pm 0.08$ & $-0.11 \pm 0.03$ & $-0.89_{-0.11}^{+0.16}$ & $1.97 \pm 0.63$ \\
\hline$\Xi_{c}^{0} \rightarrow \Lambda^{0} K^{* 0}$ & $0.28 \pm 0.13$ & $-0.35 \pm 0.31$ & $-0.58_{-0.42}^{+0.51}$ & $14.64 \pm 13.20$ \\
\hline$\Xi_{c}^{0} \rightarrow p \rho^{-}$ & $0.15 \pm 0.11$ & $-0.58 \pm 0.25$ & $-0.92_{-0.08}^{+0.42}$ & $5.09 \pm 5.74$ \\
\hline$\Xi_{c}^{0} \rightarrow n \rho^{0}$ & $0.07 \pm 0.06$ & $-0.58 \pm 0.25$ & $-0.92_{-0.08}^{+0.08}$ & $5.08 \pm 5.74$ \\
\hline$\Xi_{c}^{0} \rightarrow n \omega$ & $0.56 \pm 0.24$ & $0.43 \pm 0.17$ & $0.69 \pm 0.28$ & $3.56 \pm 0.82$ \\
\hline$\Xi_{c}^{0} \rightarrow n \phi$ & $0.77 \pm 0.13$ & $-0.40 \pm 0.01$ & $-0.93 \pm 0.03$ & $3.77 \pm 1.04$ \\
\hline$\Xi_{c}^{0} \rightarrow \Sigma^{0} K^{* 0}$ & $0.07 \pm 0.01$ & $-0.12 \pm 0.03$ & $-0.89_{-0.11}^{+0.16}$ & $2.03 \pm 0.64$ \\
\hline$\Xi_{c}^{0} \rightarrow \Sigma^{-} K^{*+}$ & $2.08 \pm 0.14$ & $-0.17 \pm 0.01$ & $-0.96 \pm 0.02$ & $1.58 \pm 0.12$ \\
\hline
\end{tabular}

$$
\begin{aligned}
\mathcal{B}\left(\Lambda_{c}^{+}\right. & \rightarrow \Sigma(1385)^{*+(0)} \pi^{0(+)}, \\
\Sigma^{*+(0)} & \left.\rightarrow \Lambda^{0} \pi^{+(0)}\right)=(1.9 \pm 0.4) \times 10^{-3},
\end{aligned}
$$

where we have taken $\mathcal{B}\left(\Lambda_{c}^{+} \rightarrow \Sigma(1385) \pi\right)=(2.2 \pm 0.4) \times$ $10^{-3}$ from our previous work [20] and $\mathcal{B}(\Sigma(1385) \rightarrow$ $\Lambda \pi)=0.87 \pm 0.01$ in PDG [2]. By subtracting these resonant contributions in $\Lambda_{c}^{+} \rightarrow \Lambda \pi^{+} \pi^{0}$, we find that

$$
\mathcal{B}\left(\Lambda_{c}^{+} \rightarrow \Lambda^{0} \pi^{0} \pi^{+}\right)_{\text {non }} / \mathcal{B}\left(\Lambda_{c}^{+} \rightarrow \Lambda \pi^{0} \pi^{+}\right)<44 \%
$$

with $90 \%$ C.L. and $\mathcal{B}\left(\Lambda_{c}^{+} \rightarrow \Lambda^{0} \pi^{0} \pi^{+}\right)_{\text {non }}=(1.9 \pm 0.7) \%$ by neglecting other resonant channels, where the subscript of "non" represents the nonresonant contribution only. This result shows that $\Lambda_{c}^{+} \rightarrow \Lambda^{0} \pi^{+} \pi^{0}$ is dominated by the resonances, which is one of the important predictions in Ref. [21].

We point out that the decays of $\Lambda_{c}^{+} \rightarrow \Sigma^{+} K^{* 0}$ and $\Xi_{c}^{+} \rightarrow$ $p \bar{K}^{* 0}$ are dominated by the $P_{1}$ waves in Eq. (10), which contribute roughly 10 times larger than those from the $S_{1}$, $P_{2}$, and $D$ waves in Eqs. (9), (11), and (12), resulting in $\alpha_{V} \approx 10$. Two decays share the same coupling strengths in terms of the $U$-spin symmetry [27], as they are related through interchanging $d$ and $s$ quarks. Naively, one expects that they should have the same decay widths. However, our results indicate that

$\Gamma\left(\Lambda_{c}^{+} \rightarrow \Sigma^{+} K^{* 0}\right) / \Gamma\left(\Xi_{c}^{+} \rightarrow p \bar{K}^{* 0}\right)_{S U(3)_{F}}=0.18 \pm 0.01$,

in which the uncertainty is very small. This hierarchy in Eq. (39) can be understood by the phase space differences. In the limit of $P_{1} \gg\left(S, P_{2}, D\right)$, we have $\Gamma \propto p_{c}^{3}+O\left(p_{c}^{5}\right)$, which leads to the suppression in $\Lambda_{c}^{+} \rightarrow \Sigma^{+} K^{* 0}$ due to the smaller released energy given by

$$
\begin{aligned}
m_{\Lambda_{c}^{+}}-m_{\Sigma^{+}}-m_{K^{* 0}} & =0.20 \mathrm{GeV}, \\
m_{\Xi_{c}^{+}}-m_{p}-m_{\bar{K}^{* 0}} & =0.64 \mathrm{GeV} .
\end{aligned}
$$

It can be interpreted as the $S U(3)_{F}$ breaking effect, caused by the mass differences. Meanwhile, the experimental data lead to

$$
\Gamma\left(\Lambda_{c}^{+} \rightarrow \Sigma^{+} K^{* 0}\right) / \Gamma\left(\Xi_{c}^{+} \rightarrow p \bar{K}^{* 0}\right)_{\mathrm{ex}}=1.9 \pm 0.8,
$$

which is much larger than the value in Eq. (39). We remark that in Ref. [25] the ratio is obtained as 0.66 , which is also smaller than the experimental value in Eq. (41). Despite this inconsistence, we are still confident that our result in Eq. (39) due to the phase space suppression is correct. We view this result as one of our predictions and suggest future experiments to revisit the channels.

It is interesting to note that the Cabibbo allowed decays of $\Lambda_{c}^{+} \rightarrow \Lambda^{0} \rho^{+}$and $\Xi_{c}^{0} \rightarrow \Xi^{-} \rho^{+}$have large branching ratios and decay parameters with small uncertainties, as shown in Table II, so that they can be viewed as the golden modes for the experimental searches. Similarly, the singly Cabibbo suppressed decays of $\Lambda_{c}^{+} \rightarrow \Lambda^{0} K^{*+}, \Xi_{c}^{+} \rightarrow \Sigma^{+} \phi$, and $\Xi_{c}^{0} \rightarrow$ $\Xi^{-} K^{*+}$ are also recommended to future experiments for the same reasons. In addition, we point out that the decay parameters in $\Xi_{c}^{+(0)} \rightarrow \Sigma^{+(0)} \phi$ are almost the same in terms of the isospin symmetry. However, the decay branching ratio for the neutral $\Xi_{c}^{0}$ mode is suppressed due to the shorter lifetime compared to the $\Xi_{c}^{+}$one and the factor 2 from the Clebsch-Gordan (CG) coefficient.

In Table $\mathrm{V}$, we compare our results of the Cabibbo allowed decays with those in the literature, where Körner and Krämer (KK) [38], Żenczykowski (Zen) [45] and 
TABLE V. Decay branching ratios (\%) of the Cabibbo favored channels in our $S U(3)_{F}$ approach and those in KK [38], Zen [45], and HYZ [25], along with the data in Ref. [2].

\begin{tabular}{|c|c|c|c|c|c|}
\hline Channel & Our results & KK [38] & Zen [45] & HYZ [25] & Data [2] \\
\hline$\Lambda_{c}^{+} \rightarrow \Lambda^{0} \rho^{+}$ & $4.81 \pm 0.58$ & 19.4 & 1.80 & $0.74 \pm 0.34$ & $<6$ \\
\hline$\Lambda_{c}^{+} \rightarrow p \bar{K}^{* 0}$ & $2.03 \pm 0.25$ & 3.13 & 5.03 & $1.9 \pm 0.3$ & $1.96 \pm 0.27$ \\
\hline$\Lambda_{c}^{+} \rightarrow \Sigma^{0} \rho^{+}$ & $1.43 \pm 0.42$ & 3.19 & 1.56 & $0.61 \pm 0.46$ & \\
\hline$\Lambda_{c}^{+} \rightarrow \Sigma^{+} \rho^{0}$ & $1.43 \pm 0.42$ & 3.17 & 1.56 & $0.61 \pm 0.46$ & \\
\hline$\Lambda_{c}^{+} \rightarrow \Sigma^{+} \omega$ & $1.81 \pm 0.19$ & 4.09 & 1.10 & $1.6 \pm 0.7$ & $1.70 \pm 0.21$ \\
\hline$\Lambda_{c}^{+} \rightarrow \Sigma^{+} \phi$ & $0.39 \pm 0.06$ & 0.26 & 0.11 & $0.39 \pm 0.06$ & $0.39 \pm 0.06$ \\
\hline$\Lambda_{c}^{+} \rightarrow \Xi^{0} K^{*+}$ & $0.10 \pm 0.10$ & 0.12 & 0.11 & $0.87 \pm 0.27$ & \\
\hline$\Xi_{c}^{+} \rightarrow \Sigma^{+} \bar{K}^{* 0}$ & $1.40 \pm 0.69$ & 2.42 & 7.38 & $10.1 \pm 2.9$ & $2.88 \pm 1.06$ \\
\hline$\Xi_{c}^{+} \rightarrow \Xi^{0} \rho^{+}$ & $14.48 \pm 2.44$ & 99.0 & 5.48 & $9.9 \pm 2.9$ & $8.2 \pm 3.6$ \\
\hline$\Xi_{c}^{0} \rightarrow \Lambda^{0} \bar{K}^{* 0}$ & $1.37 \pm 0.26$ & 1.55 & 1.15 & $0.46 \pm 0.21$ & \\
\hline$\Xi_{c}^{0} \rightarrow \Sigma^{0} \bar{K}^{* 0}$ & $0.42 \pm 0.23$ & 0.85 & 0.77 & $0.27 \pm 0.22$ & \\
\hline$\Xi_{c}^{0} \rightarrow \Sigma^{+} K^{*-}$ & $0.24 \pm 0.17$ & 0.54 & 0.37 & $0.93 \pm 0.29$ & \\
\hline$\Xi_{c}^{0} \rightarrow \Xi^{0} \rho^{0}$ & $0.88 \pm 0.22$ & 2.36 & 1.22 & $1.4 \pm 0.4$ & \\
\hline$\Xi_{c}^{0} \rightarrow \Xi^{0} \omega$ & $2.78 \pm 0.45$ & 3.21 & 0.15 & $0.10_{-0.10}^{+0.86}$ & \\
\hline$\Xi_{c}^{0} \rightarrow \Xi^{0} \phi$ & $0.14 \pm 0.13$ & 0.25 & 0.10 & $0.015_{-0.015}^{+0.071}$ & \\
\hline$\Xi_{c}^{0} \rightarrow \Xi^{-} \rho^{+}$ & $8.98 \pm 0.55$ & 16.9 & 1.50 & $0.86 \pm 0.12$ & \\
\hline
\end{tabular}

Hsiao et al. (HYZ) [25] are the studies based on the covariant quark model, pole model, and $S U(3)_{F}$, respectively. In Ref. [38], only the decay widths are provided, instead of the branching ratios. To obtain the branching ratios, we have used the lifetimes in Eq. (1). As seen from Table V, our results are consistent with those in Ref. [38]. However, the branching ratios of $\rho^{+}$modes of $\Lambda_{c}^{+} \rightarrow \Lambda^{0} \rho^{+}$, $\Lambda_{c}^{+} \rightarrow \Sigma^{0} \rho^{+}, \Xi_{c}^{+} \rightarrow \Xi^{0} \rho^{+}$, and $\Xi_{c}^{0} \rightarrow \Xi^{-} \rho^{+}$in Ref. [38] are too large compared to our predictions as well as the existing data. Furthermore, most of our results are compatible with those in Ref. [45], whereas they differ largely in $\Lambda_{c}^{+} \rightarrow \Lambda^{0} \rho^{+}, \Xi_{c}^{0} \rightarrow\left(\Xi^{-} \rho^{+}, \Xi^{0} \omega\right)$, and $\Xi_{c}^{+}$decays. Note that, in Ref. [25], the contributions from $H(\overline{15})$ and the correlations between the parameters are not included in their calculations, resulting in larger errors than ours. Except for the decays with the existing experimental data, which are also the inputs for the fitting, the predictions in Ref. [25] are quite different from ours, even though both of us take the $S U(3)_{F}$ approach. In particular, due to the different treatments of the wave amplitude, the predicted decay branching ratio of $\Lambda_{c}^{+} \rightarrow \Xi^{0} K^{*+}$ in Ref. [25] is about 8 times larger than ours and the one in the literature $[38,45]$.

\section{CONCLUSIONS}

We have explored the charmed baryon decays of $\mathbf{B}_{c} \rightarrow \mathbf{B}_{n} V$ based on the $S U(3)_{F}$ flavor symmetry. In these processes, we have calculated the color-symmetric parts of the effective Hamiltonian by the factorization approach assisted with the MIT bag model, while the antisymmetric ones are extracted from the experimental data. We have systematically obtained all decay branching ratios and parameters in $\mathbf{B}_{c} \rightarrow \mathbf{B}_{n} V$. We have found that our results are consistent with the experimental data except $\Lambda_{c}^{+} \rightarrow \Sigma^{0} K^{*+}$, for which our fitted value of $\mathcal{B}\left(\Lambda_{c}^{+} \rightarrow \Sigma^{0} K^{*+}\right)=(0.38 \pm 0.09) \times 10^{-3}$ is much smaller than the data of $(3.5 \pm 1.0) \times 10^{-3}$. As our result contains a very small error, whereas the experimental one is large, we are eager to see the precision measurement of this mode in future experiments. We have demonstrated that the branching ratios of $\Lambda_{c}^{+} \rightarrow \Lambda^{0} \pi^{+} \pi^{0}$ and $\Xi_{c}^{+} \rightarrow$ $\Xi^{0} \pi^{+} \pi^{0}$ are dominated by the resonances with the decay chains of $\Lambda_{c}^{+} \rightarrow \Lambda^{0} \rho^{+}, \rho^{+} \rightarrow \pi^{+} \pi^{0}$, and $\Xi_{c}^{+} \rightarrow \Xi^{0} \rho^{+}$, $\rho^{+} \rightarrow \pi^{+} \pi^{0}$, respectively. We have shown that most of our results with $S U(3)_{F}$ are consistent with those calculated from the dynamical models in Refs. $[38,45]$. However, the predictions for the $\rho^{+}$modes of $\Lambda_{c}^{+} \rightarrow \Lambda^{0} \rho^{+}, \Lambda_{c}^{+} \rightarrow \Sigma^{0} \rho^{+}$, $\Xi_{c}^{+} \rightarrow \Xi^{0} \rho^{+}$, and $\Xi_{c}^{0} \rightarrow \Xi^{-} \rho^{+}$in Ref. [38] are too large, whereas those of $\Lambda_{c}^{+} \rightarrow \Lambda^{0} \rho^{+}$and $\Xi_{c}^{0} \rightarrow\left(\Xi^{-} \rho^{+}, \Xi^{0} \omega\right)$ in Ref. [45] are found to be too small, compared to our values. On the other hand, our results are very different from those in Ref. [25], in which the $S U(3)_{F}$ approach is also used, but the contributions from color-symmetric parts of the effective Hamiltonian are ignored.

\section{ACKNOWLEDGMENTS}

We thank Professor Hai-Bo Yu for useful discussions. This work was supported in part by National Center for Theoretical Sciences and MoST (MoST-107-2119-M-007-013-MY3).

\section{APPENDIX A: DYNAMICS}

To get a consistent results with the $S U(3)_{F}$ representation in Sec. II, we adopt the baryon wave functions as

$$
\mathbf{B}_{c}=\frac{1}{\sqrt{6}}\left[\left(\mathbf{B}_{c}\right)_{k} \epsilon^{i j k} q_{i} q_{j} c \otimes \chi_{A}+(23)+(13)\right]
$$


TABLE VI. Form factors for charmed baryons decaying to octet baryons with $q^{2}=0$.

\begin{tabular}{lrrrrrr}
\hline \hline Channel & \multicolumn{1}{c}{$f_{1}$} & \multicolumn{1}{c}{$f_{2}$} & \multicolumn{1}{c}{$f_{3}$} & \multicolumn{1}{c}{$g_{1}$} & \multicolumn{1}{c}{$g_{2}$} & \multicolumn{1}{c}{$g_{3}$} \\
\hline$\Lambda_{c}^{+} \rightarrow \Lambda^{0}$ & -0.455 & -0.189 & -0.001 & -0.497 & 0.055 & 0.438 \\
$\Lambda_{c}^{+} \rightarrow p$ & 0.328 & 0.181 & 0.000 & 0.407 & -0.070 & -0.501 \\
$\Lambda_{c}^{+} \rightarrow n$ & 0.330 & 0.182 & -0.000 & 0.408 & -0.070 & -0.502 \\
$\Xi_{c}^{+} \rightarrow \Lambda^{0}$ & -0.138 & -0.093 & 0.009 & -0.168 & 0.026 & 0.271 \\
$\Xi_{c}^{+} \rightarrow \Sigma^{0}$ & 0.290 & 0.201 & -0.031 & 0.332 & -0.031 & -0.550 \\
$\Xi_{c}^{+} \rightarrow \Sigma^{+}$ & -0.410 & -0.285 & 0.044 & -0.469 & 0.044 & 0.778 \\
$\Xi_{c}^{+} \rightarrow \Xi^{0}$ & -0.587 & -0.309 & 0.029 & -0.630 & 0.053 & 0.732 \\
$\Xi_{c}^{0} \rightarrow \Lambda^{0}$ & 0.137 & 0.093 & -0.009 & 0.167 & -0.026 & -0.271 \\
$\Xi_{c}^{0} \rightarrow \Sigma^{0}$ & 0.288 & 0.201 & -0.031 & 0.330 & -0.031 & -0.549 \\
$\Xi_{c}^{0} \rightarrow \Sigma^{-}$ & 0.408 & 0.284 & -0.044 & 0.467 & -0.045 & -0.777 \\
$\Xi_{c}^{0} \rightarrow \Xi^{-}$ & 0.590 & 0.312 & -0.030 & 0.632 & -0.052 & -0.738 \\
\hline \hline
\end{tabular}

and

$$
\mathbf{B}_{n}=\frac{1}{3}\left[\left(\mathbf{B}_{n}\right)_{l}^{i} \epsilon^{l j k} q_{j} q_{k} q_{i} \otimes \chi_{A}+(23)+(13)\right]
$$

for the antitriplet charmed and octet baryons, respectively, where (23) stands for interchanging second and third quarks in the first term, while (13) stands for first and third ones. Here, the spin structure is defined as $\chi_{A}=$ $(\uparrow \downarrow \uparrow-\downarrow \uparrow \uparrow) / \sqrt{2}$.

The definitions in Eqs. (A1) and (A2) have different signs for $\Xi^{-}$and $\Lambda_{c}^{+}$compared to those in Refs. [43,44], while they differ in sign for $\Sigma^{+}, \Xi^{0}$, and $\Lambda^{0}$ in Ref. [56].

In this work, the form factors are evaluated from the MIT bag model [55,56]. We follow the calculations in Ref. [56]. For completeness, the input parameters are given by

$$
\begin{aligned}
m_{u, d} & =0.005 \mathrm{GeV}, \quad m_{s}=0.28 \mathrm{GeV}, \\
m_{c} & =1.5 \mathrm{GeV}, \quad R=5 \mathrm{GeV}^{-1}
\end{aligned}
$$

where $R$ is the radius of the quark bag. After correcting a typographical error in the original derivation, Eq. (19f) in Ref. [56] shall be read as

$$
\mathcal{A}_{T}=(A-B) N^{i} N^{f} R^{3} W_{-}^{i} W_{-}^{f} J_{11}\left(-2 R^{2} / 15\right),
$$

where $\mathcal{A}_{T}$ is one of the components in the axial vector, $A(B)$ is the quark overlapping factor for the spin-up (down), $N^{i}\left(N^{f}\right)$ is the normalized factor for the initial (final) baryon, $W_{-}^{i(f)}$ is associated with the normalized factor for quarks, and $J_{11}$ is the overlapping between two Bessel functions. The details can be found in Ref. [56].

Our results with $q^{2}=0$ are provided in Table VI, where we have assumed the dipole momentum dependences, given by

$$
f_{i}=\frac{f(0)}{\left(1-\frac{q^{2}}{M_{V}^{2}}\right)^{2}}, \quad g_{i}=\frac{g(0)}{\left(1-\frac{q^{2}}{M_{A}^{2}}\right)^{2}},
$$

with $\left(M_{V}, M_{A}\right)=(2.01,2.42) \mathrm{GeV}$ for $c \rightarrow s$ and $\left(M_{V}, M_{A}\right)=(2.11,2.51) \mathrm{GeV}$ for $c \rightarrow u / d$. The sign differences in the form factors compared to Ref. [56] are due to the baryon wave functions.

\section{APPENDIX B: AMPLITUDES WITH $S U(3)_{F}$ REPRESENTATIONS}

In this Appendix, we provide the effective coupling strengths in Tables VII-IX. We distinguish $A_{1}\left(B_{1}\right)$ in two different parts according to the effective Hamiltonian.

TABLE VII. Effective coupling strengths for Cabibbo allow decays with units $10^{-1} G_{F} \mathrm{GeV}^{2}$.

\begin{tabular}{lccccc}
\hline \hline Channel & $A_{1}^{(\overline{15})}$ & $A_{2}^{(\mathrm{fac})}$ & $B_{1}^{(15)}$ & $B_{2}^{(\mathrm{fac})}$ & $A_{1}^{(6)}$ \\
\hline$\Lambda_{c}^{+} \rightarrow \Lambda^{0} \rho^{+}$ & 0.281 & 0.084 & -0.430 & 0.312 & $-\frac{\sqrt{6} a_{1}}{3}-\frac{\sqrt{6} a_{2}}{3}-\frac{\sqrt{6} a_{3}}{3}$ \\
$\Lambda_{c}^{+} \rightarrow p \bar{K}^{* 0}$ & -0.305 & 0.052 & 0.460 & 0.154 & $-2 a_{1}$ \\
$\Lambda_{c}^{+} \rightarrow \Sigma^{0} \rho^{+}$ & 0 & 0 & 0 & 0 & $-\sqrt{2} a_{1}+\sqrt{2} a_{2}+\sqrt{2} a_{3}$ \\
$\Lambda_{c}^{+} \rightarrow \Sigma^{+} \rho^{0}$ & 0 & 0 & 0 & 0 & $\sqrt{2} a_{1}-\sqrt{2} a_{2}-\sqrt{2} a_{3}$ \\
$\Lambda_{c}^{+} \rightarrow \Sigma^{+} \omega$ & 0 & 0 & 0 & 0 & $-2 \sqrt{2} \tilde{a}-\frac{\sqrt{2} a_{1}}{3}-\frac{\sqrt{2} a_{2}}{3}+\frac{\sqrt{2} a_{3}}{3}$ \\
$\Lambda_{c}^{+} \rightarrow \Sigma^{+} \phi$ & 0 & 0 & 0 & 0 & $-2 \tilde{a}+\frac{2 a_{1}}{3}+\frac{2 a_{2}}{3}-\frac{2 a_{3}}{3}$ \\
$\Lambda_{c}^{+} \rightarrow \Xi^{0} K^{*+}$ & 0 & 0 & 0 & 0 & $-2 a_{2}$ \\
$\Xi_{c}^{+} \rightarrow \Sigma^{+} \bar{K}^{* 0}$ & 0.335 & -0.030 & -0.656 & -0.224 & $2 a_{3}$ \\
$\Xi_{c}^{+} \rightarrow \Xi^{0} \rho^{+}$ & 0.350 & 0.074 & -0.619 & 0.472 & $-2 a_{3}$ \\
$\Xi_{c}^{0} \rightarrow \Lambda^{0} \bar{K}^{* 0}$ & -0.123 & 0.018 & 0.213 & 0.073 & $-\frac{2 \sqrt{6} a_{1}}{3}+\frac{\sqrt{6} a_{2}}{3}+\frac{\sqrt{6} a_{3}}{3}$ \\
$\Xi_{c}^{0} \rightarrow \Sigma^{0} \bar{K}^{* 0}$ & -0.236 & 0.021 & 0.461 & 0.158 & $-\sqrt{2} a_{2}-\sqrt{2} a_{3}$ \\
$\Xi_{c}^{0} \rightarrow \Sigma^{+} K^{*-}$ & 0 & 0 & 0 & 0 & $2 a_{2}$ \\
$\Xi_{c}^{0} \rightarrow \Xi^{0} \rho^{0}$ & 0 & 0 & 0 & 0 & $-\sqrt{2} a_{1}+\sqrt{2} a_{3}$ \\
$\Xi_{c}^{0} \rightarrow \Xi^{0} \omega$ & 0 & 0 & 0 & 0 & $\sqrt{2} a_{1}$ \\
$\Xi_{c}^{0} \rightarrow \Xi^{0} \phi$ & 0 & 0 & 0 & 0 & $2 \sqrt{2} a_{2}$ \\
3 & $0 . \frac{\sqrt{2} a_{3}}{3}$ \\
$\Xi_{c}^{0} \rightarrow \Xi^{-} \rho^{+}$ & -0.351 & -0.073 & 0.624 & -0.477 & $2 \tilde{a}-\frac{2 a_{1}}{3}+\frac{4 a_{2}}{3}+\frac{2 a_{3}}{3}$ \\
\hline \hline
\end{tabular}


TABLE VIII. Effective coupling strengths for the singly Cabibbo suppressed decays with units $10^{-2} G_{F} \mathrm{GeV}^{2}$.

\begin{tabular}{|c|c|c|c|c|c|}
\hline Channel & $A_{1}^{(\overline{15})}$ & $A_{2}^{(\mathrm{fac})}$ & $B_{1}^{(\overline{15})}$ & $B_{2}^{(\mathrm{fac})}$ & $s_{c}^{-1} A_{1}^{(6)}$ \\
\hline$\Lambda_{c}^{+} \rightarrow \Lambda^{0} K^{*+}$ & 0.812 & 0.242 & -1.291 & 0.937 & $-\frac{\sqrt{6} a_{1}}{3}+\frac{2 \sqrt{6} a_{2}}{3}-\frac{\sqrt{6} a_{3}}{3}$ \\
\hline$\Lambda_{c}^{+} \rightarrow p \rho^{0}$ & -0.397 & 0.067 & 0.574 & 0.192 & $-\sqrt{2} a_{2}-\sqrt{2} a_{3}$ \\
\hline$\Lambda_{c}^{+} \rightarrow p \omega$ & 0.406 & -0.069 & -0.589 & -0.197 & $-2 \sqrt{2} \tilde{a}+\frac{2 \sqrt{2} a_{1}}{3}-\frac{\sqrt{2} a_{2}}{3}+\frac{\sqrt{2} a_{3}}{3}$ \\
\hline$\Lambda_{c}^{+} \rightarrow p \phi$ & -0.889 & 0.150 & 1.420 & 0.476 & $-2 \tilde{a}-\frac{4 a_{1}}{3}+\frac{2 a_{2}}{3}-\frac{2 a_{3}}{3}$ \\
\hline$\Lambda_{c}^{+} \rightarrow n \rho^{+}$ & 0.563 & 0.248 & -0.815 & 0.716 & $-2 a_{2}-2 a_{3}$ \\
\hline$\Lambda_{c}^{+} \rightarrow \Sigma^{0} K^{*+}$ & 0 & 0 & 0 & 0 & $-\sqrt{2} a_{1}+\sqrt{2} a_{3}$ \\
\hline$\Lambda_{c}^{+} \rightarrow \Sigma^{+} K^{* 0}$ & 0 & 0 & 0 & 0 & $-2 a_{1}+2 a_{3}$ \\
\hline$\Xi_{c}^{+} \rightarrow \Lambda^{0} \rho^{+}$ & -0.228 & -0.085 & 0.379 & -0.339 & $-\frac{\sqrt{6} a_{1}}{3}-\frac{\sqrt{6} a_{2}}{3}+\frac{2 \sqrt{6} a_{3}}{3}$ \\
\hline$\Xi_{c}^{+} \rightarrow p \bar{K}^{* 0}$ & 0 & 0 & 0 & 0 & $-2 a_{1}+2 a_{3}$ \\
\hline$\Xi_{c}^{+} \rightarrow \Sigma^{0} \rho^{+}$ & 0.436 & 0.102 & -0.818 & 0.733 & $-\sqrt{2} a_{1}+\sqrt{2} a_{2}$ \\
\hline$\Xi_{c}^{+} \rightarrow \Sigma^{+} \rho^{0}$ & 0.436 & -0.039 & -0.818 & -0.280 & $\sqrt{2} a_{1}-\sqrt{2} a_{2}$ \\
\hline$\Xi_{c}^{+} \rightarrow \Sigma^{+} \omega$ & -0.446 & 0.040 & 0.840 & 0.287 & $-2 \sqrt{2} \tilde{a}-\frac{\sqrt{2} a_{1}}{3}-\frac{\sqrt{2} a_{2}}{3}-\frac{2 \sqrt{2} a_{3}}{3}$ \\
\hline$\Xi_{c}^{+} \rightarrow \Sigma^{+} \phi$ & 0.975 & -0.087 & -2.025 & -0.692 & $-2 \tilde{a}+\frac{2 a_{1}}{3}+\frac{2 a_{2}}{3}+\frac{4 a_{3}}{3}$ \\
\hline$\Xi_{c}^{+} \rightarrow \Xi^{0} K^{*+}$ & 1.011 & 0.215 & -1.858 & 1.418 & $-2 a_{2}-2 a_{3}$ \\
\hline$\Xi_{c}^{0} \rightarrow \Lambda^{0} \rho^{0}$ & -0.161 & 0.023 & 0.266 & 0.091 & $\frac{\sqrt{3} a_{1}}{3}+\frac{\sqrt{3} a_{2}}{3}-\frac{2 \sqrt{3} a_{3}}{3}$ \\
\hline$\Xi_{c}^{0} \rightarrow \Lambda^{0} \omega$ & 0.164 & -0.024 & -0.273 & -0.093 & $-2 \sqrt{3} \tilde{a}+\frac{\sqrt{3} a_{1}}{3}+\frac{\sqrt{3} a_{2}}{3}$ \\
\hline$\Xi_{c}^{0} \rightarrow \Lambda^{0} \phi$ & -0.359 & 0.051 & 0.658 & 0.225 & $-\sqrt{6} \tilde{a}-\frac{\sqrt{6} a_{1}}{3}-\frac{\sqrt{6} a_{2}}{3}$ \\
\hline$\Xi_{c}^{0} \rightarrow p K^{*-}$ & 0 & 0 & 0 & 0 & $2 a_{2}$ \\
\hline$\Xi_{c}^{0} \rightarrow n \bar{K}^{* 0}$ & 0 & 0 & 0 & 0 & $-2 a_{1}+2 a_{2}+2 a_{3}$ \\
\hline$\Xi_{c}^{0} \rightarrow \Sigma^{0} \rho^{0}$ & -0.307 & 0.028 & 0.575 & 0.197 & $-a_{1}-a_{2}$ \\
\hline$\Xi_{c}^{0} \rightarrow \Sigma^{0} \omega$ & 0.314 & -0.028 & -0.591 & -0.202 & $2 \tilde{a}+\frac{a_{1}}{3}+\frac{a_{2}}{3}+\frac{2 a_{3}}{3}$ \\
\hline$\Xi_{c}^{0} \rightarrow \Sigma^{0} \phi$ & -0.687 & 0.062 & 1.423 & 0.486 & $\sqrt{2} \tilde{a}-\frac{\sqrt{2} a_{1}}{3}-\frac{\sqrt{2} a_{2}}{3}-\frac{2 \sqrt{2} a_{3}}{3}$ \\
\hline$\Xi_{c}^{0} \rightarrow \Sigma^{+} \rho^{-}$ & 0 & 0 & 0 & 0 & $-2 a_{2}$ \\
\hline$\Xi_{c}^{0} \rightarrow \Sigma^{-} \rho^{+}$ & 0.614 & 0.146 & -1.151 & 1.031 & $-2 a_{1}$ \\
\hline$\Xi_{c}^{0} \rightarrow \Xi^{0} K^{* 0}$ & 0 & 0 & 0 & 0 & $2 a_{1}-2 a_{2}-2 a_{3}$ \\
\hline$\Xi_{c}^{0} \rightarrow \Xi^{-} K^{*+}$ & -1.014 & -0.210 & 1.873 & -1.431 & $2 a_{1}$ \\
\hline
\end{tabular}

TABLE IX. Effective coupling strengths for the doubly Cabibbo suppressed decays with units $10^{-3} G_{F} \mathrm{GeV}^{2}$.

\begin{tabular}{|c|c|c|c|c|c|}
\hline Channel & $A_{1}^{(\overline{15})}$ & $A_{2}^{(\mathrm{fac})}$ & $B_{1}^{(\overline{15})}$ & $B_{2}^{(\mathrm{fac})}$ & $s_{c}^{-2} A_{1}^{(6)}$ \\
\hline$\Lambda_{c}^{+} \rightarrow p K^{* 0}$ & 1.623 & -0.275 & -2.449 & -0.820 & \multirow{9}{*}{$\begin{array}{c}-\frac{\sqrt{6} a_{1}}{3}+\frac{2 \sqrt{6} a_{2}}{3}+\frac{2 \sqrt{6} a_{3}}{3} \\
-\sqrt{2} a_{2} \\
-2 \sqrt{2} \tilde{a}+\frac{2 \sqrt{2} a_{1}}{3}-\frac{\sqrt{2} a_{2}}{3}-\frac{2 \sqrt{2} a_{3}}{3} \\
-2 \tilde{a}-\frac{4 a_{1}}{3}+\frac{2 a_{2}}{3}+\frac{4 a_{3}}{3} \\
-2 a_{2} \\
-\sqrt{2} a_{1} \\
-2 a_{1}\end{array}$} \\
\hline$\Lambda_{c}^{+} \rightarrow n K^{*+}$ & 1.638 & 0.723 & -2.481 & 2.179 & \\
\hline$\Xi_{c}^{+} \rightarrow \Lambda^{0} K^{*+}$ & -0.664 & -0.246 & 1.154 & -1.031 & \\
\hline$\Xi_{c}^{+} \rightarrow p \rho^{0}$ & 0 & 0 & 0 & 0 & \\
\hline$\Xi_{c}^{+} \rightarrow p \omega$ & 0 & 0 & 0 & 0 & \\
\hline$\Xi_{c}^{+} \rightarrow p \phi$ & 0 & 0 & 0 & 0 & \\
\hline$\Xi_{c}^{+} \rightarrow n \rho^{+}$ & 0 & 0 & 0 & 0 & \\
\hline$\Xi_{c}^{+} \rightarrow \Sigma^{0} K^{*+}$ & 1.268 & 0.296 & -2.491 & 2.232 & \\
\hline$\Xi_{c}^{+} \rightarrow \Sigma^{+} K^{* 0}$ & -1.781 & 0.159 & 3.492 & 1.194 & \\
\hline$\Xi_{c}^{0} \rightarrow \Lambda^{0} K^{* 0}$ & 0.657 & -0.094 & -1.136 & -0.387 & \multirow{7}{*}{$\begin{array}{c}-\frac{\sqrt{6} a_{1}}{3}+\frac{2 \sqrt{6} a_{2}}{3}+\frac{2 \sqrt{6} a_{3}}{3} \\
-2 a_{2} \\
\sqrt{2} a_{2} \\
-2 \sqrt{2} \tilde{a}+\frac{2 \sqrt{2} a_{1}}{3}-\frac{\sqrt{2} a_{2}}{3}-\frac{2 \sqrt{2} a_{3}}{3} \\
-2 \tilde{a}-\frac{4 a_{1}}{3}+\frac{2 a_{2}}{3}+\frac{4 a_{3}}{3} \\
\sqrt{2} a_{1} \\
-2 a_{1}\end{array}$} \\
\hline$\Xi_{c}^{0} \rightarrow p \rho^{-}$ & 0 & 0 & 0 & 0 & \\
\hline$\Xi_{c}^{0} \rightarrow n \rho^{0}$ & 0 & 0 & 0 & 0 & \\
\hline$\Xi_{c}^{0} \rightarrow n \omega$ & 0 & 0 & 0 & 0 & \\
\hline$\Xi_{c}^{0} \rightarrow n \phi$ & 0 & 0 & 0 & 0 & \\
\hline$\Xi_{c}^{0} \rightarrow \Sigma^{0} K^{* 0}$ & 1.255 & -0.114 & -2.454 & -0.839 & \\
\hline$\Xi_{c}^{0} \rightarrow \Sigma^{-} K^{*+}$ & 1.788 & 0.425 & -3.502 & 3.138 & \\
\hline
\end{tabular}


$A_{1}^{(\overline{15})}$ and $B_{1}^{(\overline{15})}$ are purely factorizable, which are calculated through the form factors. On the other hand, $A_{1}^{(6)}$ is parametrized by the $S U(3)_{F}$ symmetry, while $B_{1}^{(6)}$ is obtained by substituting $b_{i}$ for $a_{i}$. The contributions from $A_{2}$ and $B_{2}$ are suppressed as implied by Eq. (5). We only consider the factorizable contributions in $A_{2}$ and $B_{2}$ for consistency with the calculation in $\Lambda_{c}^{+} \rightarrow p \phi$.

\section{APPENDIX C: EXPERIMENTAL BRANCHING RATIOS} by

In Refs. $[5,6]$, the experimental decay widths are given

$$
\begin{aligned}
& \Gamma\left(B^{-} \rightarrow \bar{\Lambda}_{c}^{-} \Xi_{c}^{0}\right)=(5.81 \pm 1.39) \times 10^{8} \mathrm{~s}^{-1}, \\
& \Gamma\left(\bar{B}^{0} \rightarrow \bar{\Lambda}_{c}^{-} \Xi_{c}^{+}\right)=(7.64 \pm 2.94) \times 10^{8} \mathrm{~s}^{-1} .
\end{aligned}
$$

The effective Hamiltonian responsible for the processes is [52]

$$
\begin{aligned}
\mathcal{H}_{\mathrm{eff}}(\Delta B=1)= & \frac{G_{F}}{\sqrt{2}}\left\{\xi_{c}\left[C_{1}(\mu) Q_{1}^{c}(\mu)+C_{2}(\mu) Q_{2}^{c}(\mu)\right]\right. \\
& +\xi_{u}\left[C_{2}(\mu) Q_{2}^{u}(\mu)+C_{2}(\mu) Q_{u}^{u}(\mu)\right] \\
& \left.-\xi_{t} \sum_{6}^{i=3} C_{i}(\mu) Q_{i}(\mu)\right\}
\end{aligned}
$$

where $\xi_{i} \equiv V_{i b}^{*} V_{i s}, C_{i}$ are the Wilson coefficients, and $O_{i}$ are given as

$$
\begin{gathered}
Q_{1}^{q}=\left(\bar{b}_{i} q_{j}\right)_{\mathrm{V}-\mathrm{A}}\left(\bar{q} j s_{i}\right)_{\mathrm{V}-\mathrm{A}}, \\
Q_{2}^{q}=(\bar{b} q)_{\mathrm{V}-\mathrm{A}}(\bar{q} s)_{\mathrm{V}-\mathrm{A}}, \\
Q_{3}=(\bar{b} s)_{\mathrm{V}-\mathrm{A}} \sum_{q}(\bar{q} q)_{\mathrm{V}-\mathrm{A}}, \\
Q_{4}=\left(\bar{b}_{i} s_{j}\right)_{\mathrm{V}-\mathrm{A}} \sum_{q}\left(\bar{q}_{j} q_{i}\right)_{\mathrm{V}-\mathrm{A}}, \\
Q_{5}=(\bar{b} s)_{\mathrm{V}-\mathrm{A}} \sum_{q}(\bar{q} q)_{\mathrm{V}+\mathrm{A}}, \\
Q_{6}=\left(\bar{b}_{i} s_{j}\right)_{\mathrm{V}-\mathrm{A}} \sum_{q}\left(\bar{q}_{j} q_{i}\right)_{\mathrm{V}+\mathrm{A}},
\end{gathered}
$$

with $q=u, d, s, c, b$ in the summations.

The tree order operators $O_{1}^{c}$ and $O_{2}^{c}$ are clearly isospin singlets since they do not contain either up or down quarks. The penguin operators $O_{3} \sim O_{6}$ are also isospin singlets, since they treat $u$ and $d$ on equal footing. By neglecting $O_{1}^{u}$ and $O_{2}^{u}$ due to $\xi_{u}<0.001$, we find that the effective Hamiltonian is an isospin scalar. By using the identity

$$
\begin{aligned}
\left\langle\frac{1}{2}\right. & ,\left.\frac{1}{2}\right|_{B}\left(|0,0\rangle \otimes\left|\frac{1}{2}, \frac{1}{2}\right\rangle\right)_{\mathbf{B}_{c}} \\
\quad & =\left\langle\frac{1}{2},-\left.\frac{1}{2}\right|_{B}\left(|0,0\rangle \otimes\left|\frac{1}{2},-\frac{1}{2}\right\rangle\right)_{\mathbf{B}_{c}},\right.
\end{aligned}
$$

with

$$
\begin{aligned}
& \left|\frac{1}{2}, \frac{1}{2}\right\rangle_{B}=-\left|\bar{B}^{0}\right\rangle, \quad\left|\frac{1}{2},-\frac{1}{2}\right\rangle_{B}=\left|B^{-}\right\rangle, \\
& \left|\frac{1}{2}, \frac{1}{2}\right\rangle_{\mathbf{B}_{c}}=\left|\Xi_{c}^{+}\right\rangle, \quad\left|\frac{1}{2},-\frac{1}{2}\right\rangle_{\mathbf{B}_{c}}=\left|\Xi_{c}^{0}\right\rangle, \\
& |0,0\rangle_{\mathbf{B}_{c}}=\left|\Lambda_{c}^{+}\right\rangle_{\mathbf{B}_{c}},
\end{aligned}
$$

we find that the two processes have the same decay widths as stated in Ref. [6].

We average the decay widths in Eqs. (C2) and (C1), given by

$$
\Gamma\left(B \rightarrow \Lambda_{c}^{-} \Xi_{c}\right)=(6.14 \pm 1.26) \times 10^{8} \mathrm{~s}^{-1},
$$

which has a small uncertainty. With $\mathcal{B}\left(\bar{B}^{0} \rightarrow \bar{\Lambda}_{c}^{-} \Xi_{c}^{+}\right) \times$ $\mathcal{B}\left(\Xi_{c}^{+} \rightarrow \Xi^{-} \pi^{+} \pi^{+}\right)=(3.32 \pm 0.81) \times 10^{-5}$ in Ref. [6], we get

$$
\mathcal{B}\left(\Xi_{c}^{+} \rightarrow \Xi^{-} \pi^{+} \pi^{+}\right)=(3.56 \pm 1.13) \% .
$$

From Eq. (C14) and the CLEO data [60], we have

$$
\mathcal{B}_{\mathrm{ex}}\left(\Xi_{c}^{+} \rightarrow \Xi^{0} \pi^{+} \pi^{0}\right)=(8.2 \pm 3.6) \%,
$$

which contains both resonant and nonresonant contributions. On the other hand, the latest $S U(3)_{F}$ analysis with the nonresonance shows that [21]

$$
\mathcal{B}_{\text {non }}\left(\Xi_{c}^{+} \rightarrow \Xi^{0} \pi^{+} \pi^{0}\right)=(1.5 \pm 0.3) \% .
$$

As a result, the experimental branching ratio is clearly dominated by the resonance. There are two dominating candidates in the resonances, $\Xi_{c}^{+} \rightarrow \Xi^{0}(1530) \pi^{+}, \Xi^{0} \rho^{+}$. However, the first process is forbidden by the color symmetry $[20,53,54]$, which is supported by the experimental data [60]

$\mathcal{B}\left(\Xi_{c}^{+} \rightarrow \Xi^{0}(1530) \pi^{+}\right) / \mathcal{B}\left(\Xi_{c}^{+} \rightarrow \Xi^{0} \pi^{+} \pi^{0}\right)<0.3$.

Consequently, we could safely treat the experimental value of $\mathcal{B}\left(\Xi_{c}^{+} \rightarrow \Xi^{0} \pi^{+} \pi^{0}\right)$ as $\mathcal{B}\left(\Xi_{c}^{+} \rightarrow \Xi^{0} \rho^{+}\right)$. 
[1] R. Aaij et al. (LHCb Collaboration), Phys. Rev. D 100, 032001 (2019).

[2] M. Tanabashi et al. (Particle Data Group), Phys. Rev. D 98, 030001 (2018).

[3] M. Ablikim et al. (BESIII Collaboration), Phys. Rev. Lett. 116, 052001 (2016).

[4] A. Zupanc et al. (Belle Collaboration), Phys. Rev. Lett. 113, 042002 (2014).

[5] Y. B. Li et al. (Belle Collaboration), Phys. Rev. Lett. 122, 082001 (2019).

[6] Y. B. Li et al. (Belle Collaboration), Phys. Rev. D 100, 031101 (2019).

[7] M. J. Savage and R. P. Springer, Phys. Rev. D 42, 1527 (1990).

[8] M. J. Savage, Phys. Lett. B 257, 414 (1991).

[9] K. K. Sharma and R. C. Verma, Phys. Rev. D 55, 7067 (1997).

[10] L. L. Chau, H. Y. Cheng, and B. Tseng, Phys. Rev. D 54, 2132 (1996).

[11] C. D. Lu, W. Wang, and F. S. Yu, Phys. Rev. D 93, 056008 (2016).

[12] C. Q. Geng, Y. K. Hsiao, Y. H. Lin, and L. L. Liu, Phys. Lett. B 776, 265 (2018).

[13] C. Q. Geng, Y. K. Hsiao, C. W. Liu, and T. H. Tsai, J. High Energy Phys. 11 (2017) 147.

[14] C. Q. Geng, Y. K. Hsiao, C. W. Liu, and T. H. Tsai, Phys. Rev. D 97, 073006 (2018).

[15] C. Q. Geng, Y. K. Hsiao, C. W. Liu, and T. H. Tsai, Eur. Phys. J. C 78, 593 (2018).

[16] C. Q. Geng, Y. K. Hsiao, C. W. Liu, and T. H. Tsai, Phys. Rev. D 99, 073003 (2019).

[17] C. Q. Geng, C. W. Liu, and T. H. Tsai, Phys. Lett. B 790, 225 (2019).

[18] C. Q. Geng, C. W. Liu, and T. H. Tsai, Phys. Lett. B 794, 19 (2019).

[19] C. Q. Geng, C. W. Liu, T. H. Tsai, and S. W. Yeh, Phys. Lett. B 792, 214 (2019).

[20] C. Q. Geng, C. W. Liu, T. H. Tsai, and Y. Yu, Phys. Rev. D 99, 114022 (2019).

[21] J. Y. Cen, C. Q. Geng, C. W. Liu, and T. H. Tsai, Eur. Phys. J. C 79, 946 (2019).

[22] H. J. Zhao, Y. K. Hsiao, and Y. Yao, J. High Energy Phys. 02 (2020) 165.

[23] D. Wang, P. F. Guo, W. H. Long, and F. S. Yu, J. High Energy Phys. 03 (2018) 066.

[24] X. G. He, Y. J. Shi, and W. Wang, arXiv:1811.03480.

[25] Y. K. Hsiao, Y. Yao, and H. J. Zhao, Phys. Lett. B 792, 35 (2019).

[26] S. Roy, R. Sinha, and N. G. Deshpande, Phys. Rev. D 101, 036018 (2020).

[27] C. P. Jia, D. Wang, and F. S. Yu, arXiv:1910.00876.

[28] M. Ablikim et al. (BESIII Collaboration), Phys. Rev. Lett. 115, 221805 (2015).

[29] M. Ablikim et al. (BESIII Collaboration), Phys. Rev. Lett. 117, 232002 (2016).
[30] M. Ablikim et al. (BESIII Collaboration), Phys. Rev. Lett. 118, 112001 (2017).

[31] M. Ablikim et al. (BESIII Collaboration), Phys. Rev. D 95, 111102 (2017).

[32] M. Ablikim et al. (BESIII Collaboration), Phys. Lett. B 767, 42 (2017).

[33] M. Ablikim et al. (BESIII Collaboration), Phys. Lett. B 772, 388 (2017).

[34] M. Ablikim et al. (BESIII Collaboration), Phys. Rev. Lett. 121, 062003 (2018).

[35] M. Ablikim et al. (BESIII Collaboration), Phys. Lett. B 783, 200 (2018).

[36] M. Ablikim et al. (BESIII Collaboration), Chin. Phys. C 43, 083002 (2019).

[37] M. Ablikim et al. (BESIII Collaboration), Phys. Rev. D 99, 032010 (2019).

[38] J. G. Körner and M. Krämer, Z. Phys. C 55, 659 (1992).

[39] Q. P. Xu and A. N. Kamal, Phys. Rev. D 46, 3836 (1992).

[40] Q. P. Xu and A. N. Kamal, Phys. Rev. D 46, 270 (1992).

[41] H. Y. Cheng and B. Tseng, Phys. Rev. D 48, 4188 (1993).

[42] H. Y. Cheng and B. Tseng, Phys. Rev. D 46, 1042 (1992); 55, 1697(E) (1997).

[43] H. Y. Cheng, X. W. Kang, and F. Xu, Phys. Rev. D 97, 074028 (2018).

[44] J. Zou, F. Xu, G. Meng, and H. Y. Cheng, Phys. Rev. D 101, 014011 (2020).

[45] P. Zenczykowski, Phys. Rev. D 50, 5787 (1994).

[46] T. Uppal, R. C. Verma, and M. P. Khanna, Phys. Rev. D 49 , 3417 (1994).

[47] M. A. Ivanov, J. G. Korner, V. E. Lyubovitskij, and A. G. Rusetsky, Phys. Rev. D 57, 5632 (1998).

[48] K. K. Sharma and R. C. Verma, Eur. Phys. J. C 7, 217 (1999).

[49] S. L. Chen, X. H. Guo, X. Q. Li, and G. L. Wang, Commun. Theor. Phys. 40, 563 (2003).

[50] W. Wang, F. S. Yu, and Z. X. Zhao, Eur. Phys. J. C 77, 781 (2017).

[51] S. Pakvasa, S. P. Rosen, and S. F. Tuan, Phys. Rev. D 42, 3746 (1990).

[52] G. Buchalla, A. J. Buras, and M. E. Lautenbacher, Rev. Mod. Phys. 68, 1125 (1996).

[53] J. G. Körner, Nucl. Phys. B25, 282 (1971).

[54] J. C. Pati and C. H. Woo, Phys. Rev. D 3, 2920 (1971).

[55] Y. Nambu and G. Jona-Lasinio, Phys. Rev. 122, 345 (1961).

[56] R. Perez-Marcial, R. Huerta, A. Garcia, and M. Avila-Aoki, Phys. Rev. D 40, 2955 (1989).

[57] J. M. Link et al. (FOCUS Collaboration), Phys. Lett. B 571, 139 (2003).

[58] Y. Kubota et al. (CLEO Collaboration), Phys. Rev. Lett. 71, 3255 (1993).

[59] P. Avery et al. (CLEO Collaboration), Phys. Lett. B 325, 257 (1994).

[60] K. W. Edwards et al. (CLEO Collaboration), Phys. Lett. B 373, 261 (1996). 